\title{
The Enhanced Performance Launcher Design For The ITER Upper Port ECH Antenna
}

\author{
M.A. Henderson ${ }^{1}$, R.Chavan ${ }^{1}$, R. Bertizzolo ${ }^{1}$, A. Bruschi ${ }^{2}$, S. Criant ${ }^{2}$, \\ J. Duron ${ }^{1}$, D. Farina ${ }^{2}$, R. Heidinger ${ }^{3}$, J-D. Landis ${ }^{1}$, E. Poli ${ }^{4}$, G. Ramponi ${ }^{2}$, \\ G. Saibene ${ }^{5}$, O. Sauter ${ }^{1}$, H. Shidara ${ }^{1}$, V.S. Udintsev ${ }^{1}$, H. Zohm ${ }^{4}$, C. Zucca ${ }^{1}$ \\ ${ }^{1}$ CRPP, EURATOM - Confédération Suisse, EPFL, CH-1015 Lausanne Switzerland \\ ${ }^{2}$ Istituto di Fisica del Plasma, EURATOM- ENEA-CNR Association, 20125 Milano, Italy \\ ${ }^{3}$ Forschungszentrum Karlsruhe, EURATOM-FZK, D-76021 Karlsruhe, Germany. \\ ${ }^{4}$ EFDA Close Support Unit, Boltzmannstrasse 2, D-85748 Garching, Germany \\ ${ }^{5}$ IPP-Garching, Max Planck-Institut für Plasmaphysik, D-85748 Garching, Germany
}

\begin{abstract}
The ITER ECH heating and current drive system delivers 24MW (170GHz), which can be directed to either the equatorial (EL) or upper (UL) port launching antennas depending on the desired physics application. The UL design uses two front steering (FS) mirror that sweep eight beams in a poloidal plane providing co-ECCD over the outer half of the plasma cross section. A novel frictionless, backlash-free steering mechanism has been developed for an increased reliability and providing a steering mirror rotation of up to $\pm 7^{\circ}\left( \pm 14^{\circ}\right.$ for RF beam). The principle aim of the UL is to stabilize the neoclassical tearing modes (NTM) and (by extending the steering range) access the $\mathrm{q}=1$ flux surface for control of the sawtooth oscillation. Extending the range of the UL can relax the EL steering range, and optimize the launcher for enhanced performance with an optimized central deposition and potential for counter ECCD. This paper will summarize the present UL design status along with the proposed design modifications to both launcher systems for enhanced performance.
\end{abstract}

Keywords: ECCD, ECH Antenna, NTM stabilisation

PACS: $52.50 . \mathrm{Sw}, 52.55 . \mathrm{Wq}$

\section{INTRODUCTION}

The Electron Cyclotron Heating and Current Drive (ECH\&CD) system ${ }^{1}$ for ITER is planned to consist of $24 \mathrm{MW}(60 \mathrm{~min})$ installed power at $170 \mathrm{GHz}$ and an additional $3 \mathrm{MW}(\sim 3 \mathrm{sec})$ at $\sim 120 \mathrm{GHz}$ for assisting in plasma breakdown. The ECH\&CD system consists of $24170 \mathrm{GHz}$ gyrotrons $(\geq 1 \mathrm{MW}), 3 \sim 120 \mathrm{GHz}$ gyrotrons, associated power supplies (not shown), evacuated $63.5 \mathrm{~mm} \mathrm{HE} \mathrm{HE}_{11}$ waveguide $(\sim 100 \mathrm{~m}$ in length) and two launching systems: one Equatorial Launcher ${ }^{2}$ (EL) or four Upper Launchers ${ }^{3}$ (UL). The ECH power can be directed to either launcher during a discharge with the choice depending on the physics application. For example, the EL is used when desiring more central deposition $\left(0.0 \leq \rho_{\psi} \leq 0.65\right)$, while the UL for off axis deposition $\left(0.64 \leq \rho_{\psi} \leq 0.93\right)$. The combination of the EL and UL provides access across nearly the entire plasma 
cross section. The reference design of both launchers uses a front steering (FS) mirror placed close to the plasma offering the largest steering range and optimized beam focusing. The $\mathrm{EL}^{2}$ has three sets of steering mirrors with 8 beams incident on each mirror and steered in a horizontal plane over the range of $20^{\circ} \leq \beta<45^{\circ}$, where $\beta$ is the beam's toroidal angle measured from a poloidal plane to the beam centre line. The UL (see figure 1) has two steering mirrors per port plug with 4 beams incident on each mirror. The beams are steered in a vertical plane over a range of $\Delta \alpha \sim 22^{\circ}$, where $\alpha$ corresponds to the angle from a horizontal plane down to the projected beam centre on to a poloidal plane. The vertical steering plane has a fixed toroidal injection angle of $\beta \sim 20^{\circ}$ relative to a poloidal plane, which offers the largest peak $\mathrm{j}_{\mathrm{CD}}$ for a maximum stabilisation efficiency for control of MHD activity such as neoclassical tearing mode $(\mathrm{NTM})^{4}$ and sawteeth ${ }^{5}$.

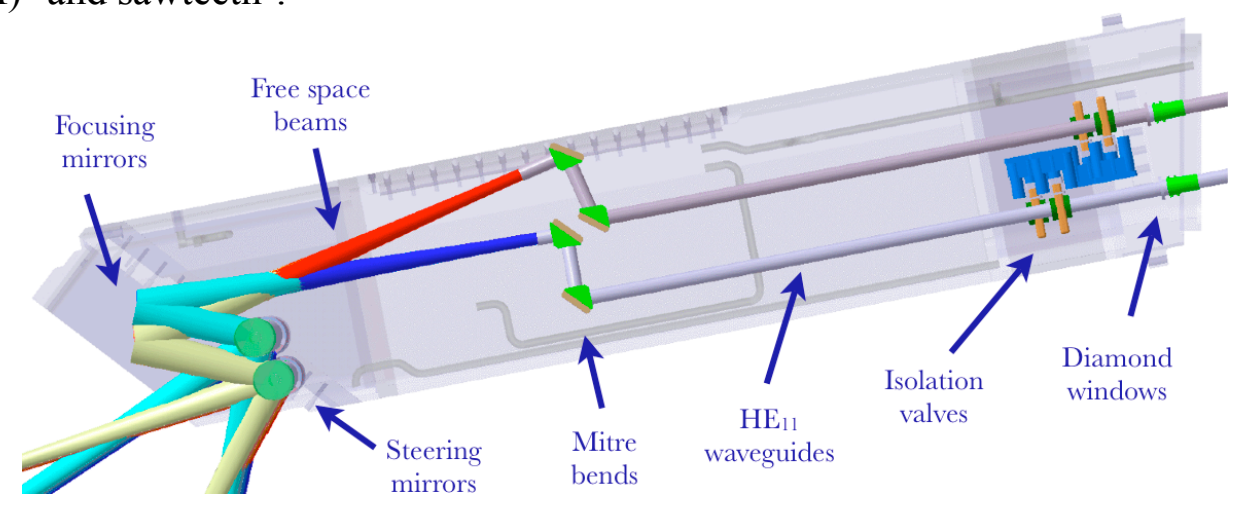

FIGURE 1. The layout of the principle components of the upper launcher.

\section{ITER Upper Launcher design}

A simplified view of the current FS launcher design is shown in Figure 1. Eight circular $\mathrm{HE}_{11}$ waveguides $\left(\phi_{\mathrm{WG}}=63.5 \mathrm{~mm}\right.$, similar to the waveguide used in the transmission line) enter the port plug entrance on the right (note that there are four waveguides in each row shown in figure 1). Prior to the closure plate a diamond window and an in-line gate or isolation valve is placed providing the primary tritium barrier. The isolation valve is on the plasma side of the diamond window, such that the window can be isolated for leak testing or repair. A miter bend 'dog-leg' assembly is used to angle the 8 beams (both in toroidal and poloidal directions) to one (or two) focusing mirror(s) with the incident beams partially overlap in both toroidal and poloidal directions. The reflected beams are then directed downward to two separate flat steering mirrors, which redirect the beams into the plasma with a toroidal injection angle of $\beta \approx 20^{\circ}$.

The present optical design of the UL is referred to as the Enhanced Performance (EP) launcher achieving a synergistic ECH system ${ }^{6}$ with the EL. The deposition region of the two UL steering mirrors are displaced so that the upper steering mirror (USM) access further inward providing access to the inner NTMs and $\mathrm{q}=1$ for control of the sawtooth oscillation covering a range of $\sim 0.4 \leq \rho_{\psi} \leq 0.89$. The lower steering mirror (LSM) access the outer NTMs covering a range of $\sim 0.75 \leq \rho_{\psi} \leq 0.93$. An additional switching system is needed prior to the port entrance that can deviate the beams 
coming from the 24 gyrotrons to either the 16 entrances associated with the USM or the 16 entrances with the LSM. The optical design is optimized ${ }^{7}$ so that the beam from the USM projects a slightly larger beam waist $(29 \mathrm{~mm})$ further into the plasma to compensate for the longer path length as compared to the LSM with a waist of $21 \mathrm{~mm}$. The free space optical design was optimized with dedicated beam tracing scans system $^{8,9}$ to insure a narrow and peaked $\mathrm{j}_{\mathrm{CD}}$ profile over the entire steering range of the launcher. Note that a single focusing and steering mirror is used for the four beams in a given row, with the beams overlapping permitting a larger beam for a finite focusing mirror size within the confined space of the blanket shield module (BSM). Note that the space in the BSM is shared between the mm-wave components and shield blocks to protect the components and port plug from the neutron flux. The focusing mirror curvature, waveguide tilt angle and relative orientation of the steering mechanism have been optimized to insure that the 4 beams are deposited nearly coincident in the plasma.

The optical design and component layout has not been finalized, for example a new design is under consideration that replaces the set of mitre bends with free space mirrors. The free space mirrors offer additional flexibility in manipulating the beam optics for improved focusing and ultimate physics performance. Also, the free space mirrors are less expensive and reduces the peak power density on the mirror surface relative to the mitre bends. A second design option is to place the isolation valves on the gyrotron side of the window, which is ITER's preferred location. This would simplify the valve design and significantly reduce the occupied volume prior to the closure plate. The present configuration has the isolation valve on the plasma side for providing in-situ window leak testing and maintenance with out effecting the torus vacuum as had been planned for the JET-EP ECH project ${ }^{10}$. These design options will be finalized during the 2007 period.

\section{Steering Mechanism design}

a)

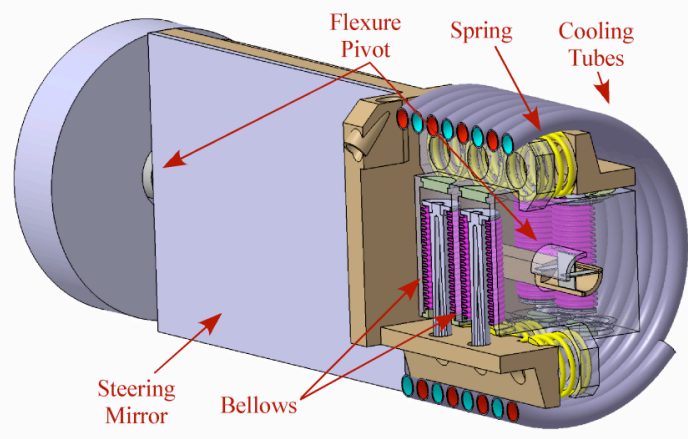

b)

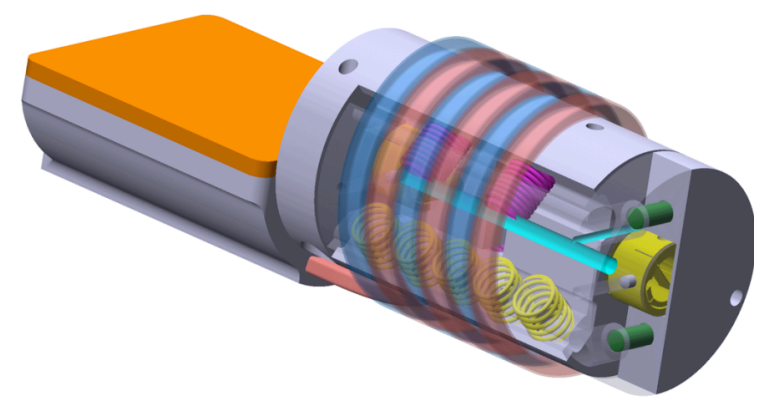

Figure 2 a) The 'balanced' and b) 'cantilevered' steering mechanism designs under consideration for the UL.

The majority of components used in the FS launcher design are similar to components that are available commercially, with the main exception being the steering mechanism ensemble. A new innovative design of the steering mechanism ${ }^{11,12}$ (see Figure 2a) is being developed that provides rotation of the steering mirror based 
on the compliant deformation of structural components offering a frictionless and backlash free mechanical movements and avoiding the invessel tribological difficulties inherent in present day FS systems. Traditional ball bearings are replaced with elastically compliant flexure pivots and the movement is controlled using an integrated gaseous helium pneumatic actuator system working against preloaded compressive springs in place of the push-pull rods. This eliminates the components that typically grip in present day FS launchers offering an improved reliability and precision in controlling the steering mirror angle. The bellows are pressurized from the outside, which offers a more stable configuration avoiding the squirm instability when the bellows are internally pressurized. A pair of coiled cooling pipes with either a single or double wall provides a flexible coolant feed to the mirror, following a similar design to that proposed for the $\mathrm{EL}^{2}$.

The steering mirror (SM) ensemble of figure $2 \mathrm{a}$ is referred to as the 'balanced SM', which has the steering mirror centered between two flexure pivots. This configuration reduces the overall forces on the flexure pivots during a vertical disruption event (VDE), but increases the risk of halo current flowing through the mirror structure and requires two supports on either side of the SM. An alternative configuration is the 'cantilevered' SM as shown in figure $2 \mathrm{~b}$, with both flexure pivots incorporated in the steering mechanism. This configuration was the original SM design back in 2003, but compatible for only two $1 \mathrm{MW}$ incident beams. The balanced SM was used to be compatible with the four $2 \mathrm{MW}$ and the additional forces associated with the larger mirror size. Optimizing the mirror design for reduced induced currents during a VDE reduces the forces on the flexure pivots, which may then allow the cantilevered SM to be reused, which simplifies the installation and repair of the SM.

\section{ACKNOWLEDGMENTS}

This work, supported by the Swiss National Science Foundation and the European Communities, was carried out within the framework of the European Fusion Development Agreement (ECHULA subtask (f) /contract EFDA TCP 341-22 and ECHULB subtask (b) /contract EFDA 05-1228). The views and opinions expressed herein do not necessarily reflect those of the European Commission.

\section{REFERENCES}

1. J. How, P. Barabaschi, W. Spears Project Integration Document, G A0 GDRD 6 04-09-09 R0.2..

2. K. Takahashi et al, Fusion Science and Technology 47 (2005) p1.

3. M.A. Henderson et al, Critical Design Issues of the ITER ECH FS Upper Launcher, accepted for publication in Fusion Science and Technology (2007).

4. S. Günter et al, Phys. Rev. Lett. 87 (2001) 275001-1.

5. J. Graves et al, Plasma Phys. Control. Fusion 47 (2005) B121-B133.

6. M. Henderson et al, Proc. of the $14^{\text {th }}$ Joint Workshop on ECE and ECRH, Santorini, Greece (2006).

7. H. Shidara et al, Proc. of the $14^{\text {th }}$ Joint Workshop on ECE and ECRH, Santorini, Greece (2006).

8. G. Ramponi et al, Journal of Physics: Conference Series 25 (2005) ) 243-251.

9. E. Poli et al, Proc. of the $14^{\text {th }}$ Joint Workshop on ECE and ECRH, Santorini, Greece (2006).

10. M.A. Henderson et al, Nucl. Fusion 251487 (2004).

11. R. Chavan et al, Journal of Physics: Conference Series 25 (2005) 151-158.

12.J.-D.Landis et al, accepted for publication in Fusion Engineering Design (2007). 